\title{
BMJ Open Perceived effectiveness of public health measures and positive attitudes during a pandemic: a large cross-sectional study in Shanghai, China
}

Qiao Chu (D) , ${ }^{1}$ Tian Gu (D) ,2 Anqi Li, ${ }^{3,4}$ Junjie Chen, ${ }^{3,4}$ Hui Wang, ${ }^{1,5}$ Ningning Liu, ${ }^{1,5}$ Ruijun Wu, ${ }^{3,4}$ Yaping $\mathrm{He}^{1,6}$

To cite: Chu Q, Gu T, Li A, et al. Perceived effectiveness of public health measures and positive attitudes during a pandemic: a large cross-sectional study in Shanghai, China. BMJ Open 2021;11:e047231. doi:10.1136/ bmjopen-2020-047231

- Prepublication history and additional supplemental material for this paper are available online. To view these files, please visit the journal online (http://dx.doi.org/10.1136/ bmjopen-2020-047231).

Received 05 December 2020 Revised 12 March 2021 Accepted 13 April 2021
Check for updates

(C) Author(s) (or their employer(s)) 2021. Re-use permitted under CC BY-NC. No commercial re-use. See rights and permissions. Published by BMJ.

For numbered affiliations see end of article.

\section{Correspondence to}

Ruijun Wu;

rjwu@re.ecnu.edu.cn and

Yaping He;

hypcyr@shsmu.edu.cn

\section{ABSTRACT}

Objectives Building individuals' positive attitudes during a pandemic is essential for facilitating psychological resilience. However, little is known about how public health measures may improve people's positive attitudes during a pandemic. We investigated the potential mechanism underlying the association between individuals' perceived effectiveness of public health measures and positive attitudes towards the success of pandemic control during the COVID-19 pandemic, by examining the parallel mediating effects of three types of threat appraisals: concerns about contracting the virus, perceived impact of the pandemic on life and estimated duration of the pandemic.

Design, setting and participants In February 2020 when the COVID-19 infection was spreading rapidly in China, a large cross-sectional survey was conducted among 132054 adults from the 16 districts in Shanghai, China. Outcome measures Perceived effectiveness of the public health measures, positive attitudes towards the success of pandemic control and threat appraisals.

Results Results of structural equation modelling supported the hypothesised mediation model: perceived effectiveness of public health measures was associated with lower levels of concerns about contracting the virus $(\beta=-0.20)$, perceived impact of the pandemic $(\beta=-0.13)$ and perceived duration of the pandemic $(\beta=-0.20)$, which were then associated with higher levels of positive attitudes towards the success of pandemic control ( $\beta s=-0.12$ to -0.25 ).

Conclusions The findings suggest that threat appraisals may be important pathways through which individuals' evaluations of prevention strategies may influence their attitudes towards the success of pandemic control. The health authorities should consider reducing people's inappropriate threat appraisals when designing public health policies to facilitate people's positive attitudes during a pandemic.

\section{INTRODUCTION}

The world is currently fighting against COVID-19. A pandemic like COVID-19 can be a stressful life event. It not only threatens individuals' health and well-being, but can also cause widespread anxiety and fear in the
Strengths and limitations of this study

- This study examined the potential psychological mechanism underlying the association between individuals' perceived effectiveness of public health measures and positive attitudes towards the success of pandemic control using a large sample recruited from the 16 districts in Shanghai.

- The present study was carried out when the fastspreading COVID-19 infection caused widespread fear and anxiety in China, and findings can inform the future implementation of public health policies to build people's positive attitudes during a pandemic.

- A theory-based parallel mediation model was tested using structural equation modelling, controlling for a variety of sociodemographic, clinical and behavioural covariates.

- Because of the social distancing policy during the study period, measures of the variables were reliant on self-reports, which may introduce response bias.

- Females, the highly educated people and younger adults were over-represented in the sample.

public. ${ }^{1}$ Building individuals' positive attitudes towards the success of pandemic control may facilitate compliance with the government's health policies, ${ }^{2}$ uptake of preventive practices $^{3}$ and psychological resilience ${ }^{4}$ during the crisis. However, research is lacking about whether people's perceptions of the public health measures can influence their attitudes towards the success of pandemic control, and the underlying psychological mechanisms.

The transactional model of stress and coping $^{56}$ suggests that individuals' responses to a stressor depend on their threat appraisals of the stressor. When confronted with a stressful event, individuals first adopt primary appraisal regarding the potential harm of the stressor. When the stressor is appraised as harmful and threatening, individuals then make secondary appraisal regarding whether they have the resources to cope with the stress. 
These appraisal processes would then influence individuals' responses to and attitudes towards the stressor. Based on the theory, people's evaluations of the effectiveness of public health measures may improve their perceived controllability of the pandemic through influencing their threat appraisals. When a pandemic is perceived as threatening to individuals' safety and well-being, they may develop negative attitudes towards the success of pandemic control. Nevertheless, effective prevention measures implemented by the health authorities may function as supportive resources ${ }^{7}$ that alleviate people's perceived harmfulness of the pandemic and may, in turn, facilitate positive attitudes and perceived controllability of the pandemic. The limited empirical studies on people's responses to public health emergencies have partially supported the theory. Studies have indicated that people's perceived effectiveness of the government's policies is associated with lower levels of perceived risks of public health emergencies. ${ }^{89}$ Another study on 9402 adults during the 2003 SARS outbreak found that participants' evaluation of the government's health policies for the pandemic was strongly associated with reduced anxiety. ${ }^{10}$ The limited empirical evidence has suggested that people's perceptions regarding public health measures are associated with their threat appraisals and responses to the pandemic. However, so far no studies have been done to examine whether threat appraisals may mediate the association between evaluations of public health measures and attitudes towards the success of pandemic control. Based on the transactional model of stress and coping, we hypothesised that individuals' perceptions regarding the effectiveness of public health measures in controlling COVID-19 would be associated with lower levels of concerns about contracting the virus, perceived impact of the pandemic on life and estimated duration of the pandemic, which were then associated with higher levels of positive attitudes towards the success of controlling COVID-19.

The present study was carried out in early February 2020, when the COVID-19 infection was spreading rapidly in China, leading to widespread fear and anxiety. As one of the municipalities in China, Shanghai faced multiple challenges in controlling the infection. First, Shanghai is a densely populated city, with 24.24 million residents as of 2018. ${ }^{11}$ This dense population puts great pressure on living supplies and healthcare resources during the pandemic, especially when travel restrictions were enforced. Second, as a major trading city and financial hub, Shanghai has attracted migrants in and outside of China, which may increase the risk of viral transmission. Third, Shanghai has an ageing population, with those over 65 years old comprising $12.93 \%$ of the population as of $2015 .^{12}$ Since the case fatality rate of COVID-19 tends to be higher among older adults, ${ }^{13}$ the large ageing population may lead to more severe cases and deaths, which may increase people's perceived threat of the pandemic and risk of psychological distress.

Since the outbreak of COVID-19 in China, the Shanghai government has implemented a variety of public health measures to control the pandemic. Social distancing policies included extending the Lunar New Year holiday, shutting down non-essential public institutions, downsizing the public transportation system and limited community access. The health authorities also set up temperature screening checkpoints at airports and railway stations, and required 14-day self-quarantine for inbound travellers. The health officials also made efforts to promote health practices, including mandatory use of face masks on public transportation, hand hygiene and cough etiquette. However, at that moment, researchers and health authorities had limited knowledge about the incubation period and modes of transmission of COVID-19. Research has demonstrated that people's evaluations of public health policies have particularly large effects on their risk appraisals when personal knowledge about the hazard is limited. ${ }^{14}$ Thus, we would expect that during this difficult time when people had little understanding about the virus, perceived effectiveness of the public health measures would serve as essential coping resources that may attenuate people's perceived threat of the pandemic, which would then facilitate positive attitudes towards the success of pandemic control.

\section{METHODS}

Patient and public involvement

No patient involved.

\section{Study design and participants}

Because of the social distancing policy to minimise in-person interactions during the study period, we administered and advertised the survey through PAPER.cn, a popular Chinese internet-based media platform. Potential participants were informed that the study aimed to understand people's life situations during the outbreak of COVID-19 in Shanghai. Shanghai residents who stayed in any of the 16 districts in Shanghai since the local outbreak (15 January 2020) were invited to participate. Patients or the public were not involved in the design, or conduct, or reporting or dissemination plans of our research. An electronic consent form was presented at the beginning of the survey. Participants need to indicate their willingness to participate by making a yes/no response. Those who indicated willingness to participate were able to take the survey. After data collection, we screened off participants who were likely not residing in Shanghai, and those residents who had left Shanghai after the outbreak, using two questions: (a) 'Since the COVID-19 outbreak (after 15 January 2020), have you left Shanghai?' and (b) 'Which district in Shanghai do you currently live in?' We retained participants who answered 'I never left Shanghai' or 'I left Shanghai after the outbreak but have now returned' to the first question, as well as those who made a valid response to the second question.

\section{MEASURES}

\section{Perceived effectiveness of public health measures}

Participants were given six major public health measures that had been implemented in Shanghai since the outbreak 
of COVID-19, including: (a) setting up body temperature screening checkpoints at the airports, railway stations and highway entrances and exits; (b) mandatory declaration of travel history and health status for incoming travellers; (c) 14-day self-quarantine for incoming travellers; (d) requiring face masks on public transportation; (e) downsizing public transportation system and (f) limited community access. Participants were asked to rate the effectiveness of each public health measure in controlling the spread of the virus using a five-point scale from 1 (not effective at all) to 5 (very effective).

\section{Positive attitudes towards the success of pandemic control}

Participants' positive attitudes were assessed using a single item 'How confident do you feel that the COVID-19 outbreak will be under control in Shanghai?' Responses were made on a five-point scale from 1 (very unconfident) to 5 (very confident).

\section{Threat appraisals}

Three types of threat appraisals were assessed: concerns about contracting the virus, perceived impact of the pandemic on life and estimated duration of the pandemic. Concerns about contracting the virus were assessed by asking 'Based on your current life and work situation, to what degree are you concerned about contracting COVID-19?' Answers were given on a four-point scale from 1 (not concerned at all) to 4 (very concerned). Perceived impact of the pandemic on life was assessed using a single item 'In general, to what extent is your life influenced by the COVID-19 outbreak?' Responses were made on a four-point scale from 1 (My life is not influenced at all) to 4 (My life is influenced a lot). Estimated duration of the outbreak was measured by asking 'How long do you think the COVID-19 outbreak will last?' Provided options included ' 1 month', ' 2 months', ' 3 months', ' 4 months or above' and 'hard to estimate'.

\section{Covariates adjusted in the mediation model}

Sociodemographic covariates included age, gender, educational level and employment status. Participants' neighbourhood was used as a proxy for socioeconomic status in the present study: low-income neighbourhoods included public housing, affordable housing and rural residence; medium-income neighbourhoods included apartment complexes in urban areas; high-income neighbourhoods included single family homes. Previous studies have indicated that poorer self-reported physical health is associated with emotional distress in reaction to COVID-19 outbreak, ${ }^{15}$ and that pre-existing chronic medical conditions predict worse outcomes of COVID19. ${ }^{16}$ Thus, individuals with chronic medical conditions or worse self-reported physical health may perceive the pandemic as more threatening. Moreover, compared with people who stayed at home most of the time during the outbreak, those who went out for work regularly may be more concerned about contracting the virus and may appraise the outbreak as more harmful. Therefore, we included self-reported physical health status (continuous variable measured using a five-point scale ranging from very unhealthy to very healthy), self-reported chronic medical conditions (having chronic medical conditions vs no chronic medical conditions) and working status during the outbreak (going out for work regularly vs working from home/on vacation/unemployed) in the analyses as additional covariates.

\section{Statistical analysis}

First, preliminary analyses were conducted in SPSS V.24.0, ${ }^{18}$ including descriptive statistics of the sample and participants' effectiveness ratings of the public health measures. Zero-order correlations were analysed among variables of interest. Continuous variables were summarised in means and SDs. Categorical variables were presented as percentages (\%).

The hypothesised parallel mediation model was tested using structural equation modelling with observed variables in Mplus V.7.0. ${ }^{19}$ Specifically, we examined the parallel mediating effects of concerns about contracting the virus, perceived impact of the pandemic on life and estimated duration of the pandemic on the association between perceived effectiveness of public health measures (averaged across the six measures) and positive attitudes towards the success of pandemic control. Adjusted covariates included age, gender, education, employment status, neighbourhoods, self-reported physical health status, selfreported chronic medical conditions and working status during the outbreak. Due to the cross-sectional design, the reverse mediation model was also tested, with individuals' positive attitudes mediating the relationship between perceived effectiveness of public health measures and threat appraisals. ${ }^{20}$

Model fit was assessed using $\chi^{2}$ goodness of fit statistic, the comparative fit index (CFI), the root mean square error of approximation (RMSEA) and the standardised root mean square residual (sRMR). A model with CFI greater than 0.95 , and with RMSEA and sRMR values less than 0.05 indicates a good fit. ${ }^{21}$ Indirect effects were assessed using 5000 bootstrapped iterations with biascorrected $95 \%$ CIs. $^{22}$ An absence of zero in the $95 \%$ CI suggests a significant indirect effect. Full information maximum likelihood (FIML) estimation was used to accommodate missing data.

\section{RESULTS}

\section{Preliminary analysis}

Among the 146086 surveys administered, 133108 valid surveys were returned (response rate=91.1\%). We excluded participants who were under 18 years old $(n=251)$ and those with missing data for age $(n=803)$, resulting in 132054 participants from the 16 districts included in the analyses. Table 1 presents the sample characteristics. Participants were between 18 and 89 years old $(\mathrm{M}=36.0, \mathrm{SD}=8.1)$. Among the enrolled participants, $72.5 \%$ were female, and $73.1 \%$ had college-level 
Table 1 Demographic characteristics of participants

Unweighted N (\%)/ mean (SD)

\begin{tabular}{|c|c|}
\hline Age (years) & $36.0(8.1)$ \\
\hline \multicolumn{2}{|l|}{ Gender } \\
\hline Male & $36346(27.5)$ \\
\hline Female & 95708 (72.5) \\
\hline \multicolumn{2}{|l|}{ Education } \\
\hline Below high school & $1155(0.9)$ \\
\hline High school & $7788(5.9)$ \\
\hline Junior college & $26601(20.1)$ \\
\hline College & $78510(59.5)$ \\
\hline Postgraduate or higher & $18000(13.6)$ \\
\hline \multicolumn{2}{|l|}{ Employment status } \\
\hline Currently employed & $120592(91.3)$ \\
\hline Retired/unemployed/homemaker & $8828(6.7)$ \\
\hline Enrolled student & $2616(2.0)$ \\
\hline Undecided & $18(<0.1)$ \\
\hline \multicolumn{2}{|l|}{ Neighbourhood } \\
\hline Low-income neighbourhood & $19830(15.0)$ \\
\hline Medium-income neighbourhood & $106572(80.7)$ \\
\hline High-income neighbourhood & $5181(3.9)$ \\
\hline Other & $471(0.4)$ \\
\hline \multicolumn{2}{|l|}{ Working status } \\
\hline Going out for work regularly & $16253(12.3)$ \\
\hline $\begin{array}{l}\text { Working from home/on vacation/ } \\
\text { unemployed }\end{array}$ & $115801(87.7)$ \\
\hline \multicolumn{2}{|l|}{$\begin{array}{l}\text { Self-reported chronic medical } \\
\text { conditions }\end{array}$} \\
\hline No chronic medical conditions & $109549(83.0)$ \\
\hline Having chronic medical conditions & $15586(11.8)$ \\
\hline Do not know & $6919(5.2)$ \\
\hline $\begin{array}{l}\text { Self-reported physical health } \\
\text { status }\end{array}$ & $4.4(0.6)$ \\
\hline
\end{tabular}

Values represent mean (SD) for age and self-reported physical health status.

n (\%) for the other variables.

education or above. The majority of participants were employed (91.3\%), and $95.7 \%$ of the participants lived in low-income or medium-income neighbourhoods. Most of the participants reported not going out to work regularly during the study period $(87.7 \%)$, and $11.8 \%$ reported having chronic medical conditions.

Table 2 presents the descriptive statistics and zero-order correlations among variables of interest. On average, participants perceived the public health measure of 'requiring face masks on public transportation' $(\mathrm{M}=4.46$, $\mathrm{SD}=0.82$ ) to be the most effective in controlling the spread of COVID-19 in Shanghai, followed by the measures of 'limited community access' $(\mathrm{M}=4.31, \mathrm{SD}=0.93)$,

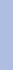

\%

Ұं

$\stackrel{m}{\sim}$

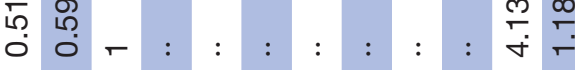

(1)

மூల ले

लं

กิ

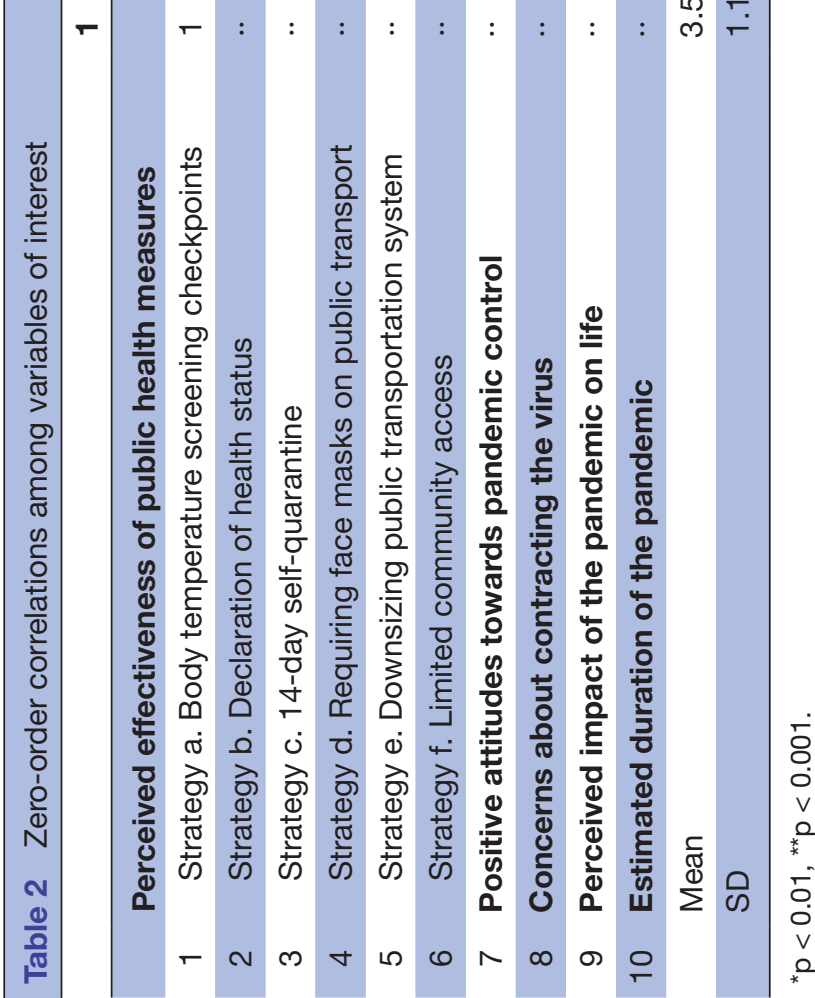




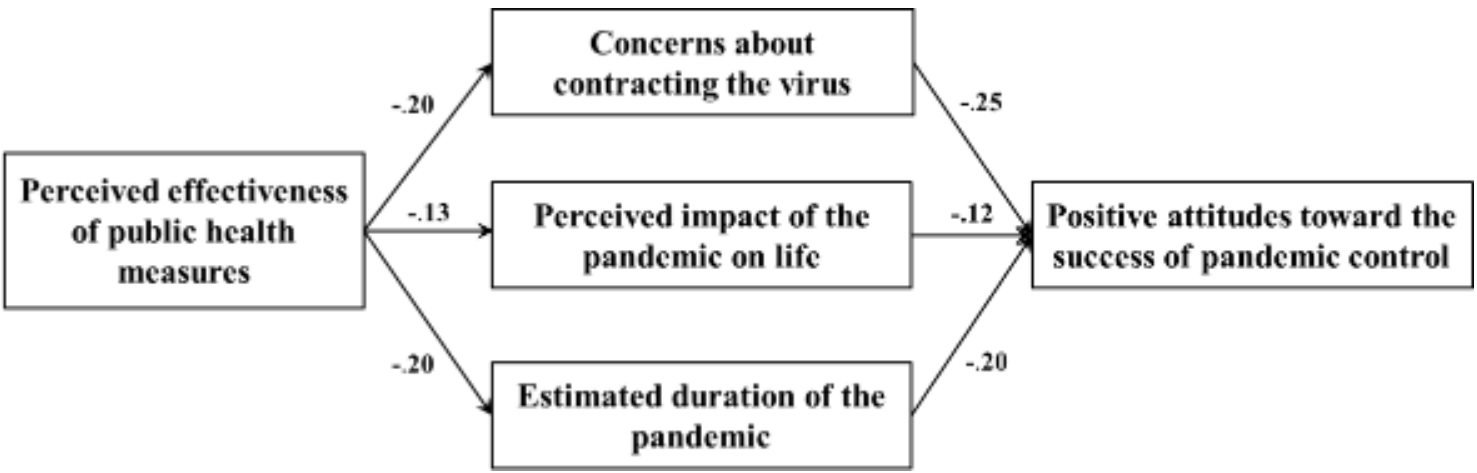

Figure 1 Hypothesised parallel mediation model. All paths shown were standardised regression coefficients, and were all significant at $p<0.001$. Adjusted covariates included sociodemographic characteristics (age, gender, educational level, employment status and neighbourhood), working status, self-reported physical health status and self-reported chronic medical condition. Correlations among the three types of threat appraisals were omitted in the figure for purpose of conciseness.

'downsizing public transportation system' $(\mathrm{M}=4.15$, $\mathrm{SD}=0.93$ ) and '14-day self-quarantine for incoming travellers' $(\mathrm{M}=4.13, \mathrm{SD}=1.18)$. Percentages of the responses for variables of interest are presented in online supplemental table S1-S5 in the Online Supplement.

Participants' effectiveness ratings for the six public health measures were associated with higher levels of positive attitudes towards the success of pandemic control ( $r=0.10$ to 0.50 ), and were associated with lower levels of perceived impact of the pandemic on life $(r=-0.01$ to -0.19 ) and estimated duration of the pandemic ( $r=-0.04$ to $-0.22)$. In addition, perceived effectiveness of public health measures was negatively associated with concerns about contracting the virus ( $\mathrm{r}=-0.04$ to -0.27$)$, except the measure of 'limited community access' $(r<0.001)$. The three types of threat appraisals were negatively associated with positive attitudes towards the success of pandemic control ( $\mathrm{r}=-0.29$ to -0.44$)$.

\section{The parallel mediation model}

The parallel mediation model yielded a good fit, $\chi^{2}$ $(3)=150.63, p<0.001, \mathrm{CFI}=0.999, \mathrm{RMSEA}=0.019,90 \% \mathrm{CI}$ (0.017 to 0.022$), \mathrm{sRMR}=0.003$. Consistent with our hypothesis, perceived effectiveness of public health measures was associated with lower levels of concerns about contracting the virus, perceived impact of the pandemic on life and perceived duration of the pandemic, which were then associated with higher levels of positive attitudes towards the success of pandemic control. Figure 1 presents the standardised model paths. Table 3 presents the specific standardised indirect effects and the $95 \%$ CIs. The total effect of perceived effectiveness of public health measures on positive attitudes (standardised total effect $=0.44$, $95 \%$ CI $(0.434$ to 0.444$), \mathrm{p}<0.001)$, total indirect effect (standardised total indirect effect $=0.11,95 \%$ CI $(0.105$ to 0.110$), \mathrm{p}<0.001$ ) and the direct effect (standardised direct effect $=0.33,95 \%$ CI $(0.33$ to 0.34$), \mathrm{p}<0.001)$ were all significant. Moreover, the specific indirect effects of perceived effectiveness of public health measures through concerns about contracting the virus, perceived impact of the pandemic and perceived duration of the pandemic were all significant. The total indirect effect through the three mediators accounted for $43.9 \%$ of the total effect. The mediation model accounted for $40.1 \%$ of the total variance of positive attitudes towards the success of pandemic control.

The reverse mediation model also indicated a good fit with the data, $\chi^{2}(1)=95.56, \mathrm{p}<0.001$, CFI $=0.999$, RMSEA $=0.027,90 \%$ CI $(0.022$ to 0.031$)$, sRMR $=0.002$. Standardised model paths are presented in online supplemental figure S1 in the Online Supplement.

Table 3 Standardised indirect effects for the parallel mediation model

\begin{tabular}{|c|c|c|}
\hline & $\begin{array}{l}\text { Standardised indirect } \\
\text { effect }\end{array}$ & $95 \% \mathrm{Cl}$ \\
\hline $\begin{array}{l}\text { Perceived effectiveness of public health measures } \\
\text { Concerns about contracting the virus } \\
\text { Positive attitudes towards pandemic control }\end{array}$ & $0.050^{\star}$ & 0.049 to 0.052 \\
\hline $\begin{array}{l}\text { Perceived effectiveness of public health measures } \\
\text { Estimated duration of the pandemic } \\
\text { Positive attitudes towards pandemic control }\end{array}$ & $0.041^{*}$ & 0.039 to 0.042 \\
\hline
\end{tabular}

${ }^{*} \mathrm{p}<0.001$ 


\section{DISCUSSION}

To the best of our knowledge, the present study is the first to investigate the potential mechanism by which individuals' perceived efficacy of public health measures may be associated with attitudes towards the success of pandemic control. There are two major findings: first, participants' effectiveness ratings varied among the six public health measures. Second, the association between perceived effectiveness of public health measures and positive attitudes was mediated by people's threat appraisals, including concerns about contracting the virus, perceived impact of the pandemic and perceived duration of the pandemic.

The results showed that participants perceived 'requiring face masks on public transportation' to be the most effective measure in controlling the spread of the virus. This may be related to people's increasing reliance on public transportation near the end of the Lunar New Year holiday. On public transportation where social distancing is difficult, it is reasonable for individuals to believe that wearing face masks would be the most effective way in avoiding virus exposure. The relatively low effectiveness rating for 'mandatory declaration of travel history and health status for incoming travellers' may be attributed to people's low trust in travellers' self-report. For the measure of 'setting up body temperature checkpoints', there are two possible explanations for its low effectiveness rating. First, by the end of January before the study was conducted, COVID-19 cases without fevers or with late presence of fevers were already reported, ${ }^{23} 24$ which may lower people's perceived effectiveness of fever screening in identifying potential patients. Second, body temperature checking may reduce mobility and cause crowding at airports, railways and subway stations, which may heighten people's concerns for viral transmission.

The present study supported the transactional model of stress and coping ${ }^{56}$ and has suggested that threat appraisals mediate the association between people's perceptions of public health policies and attitudes towards the success of pandemic control. In line with the theory, perceived effectiveness of public health measures may function as supportive resources for individuals to cope with the stress induced by the pandemic, and alleviate their perceived harmfulness of the pandemic, including concerns about contracting the virus, perceived impact of the pandemic and perceived duration of the pandemic. The reduction in perceived threat may, in turn, contribute to people's positive attitudes towards the success of pandemic control.

Our findings align with the limited quantitative studies demonstrating that individuals' evaluations of the government's health policies are associated with threat appraisals of public health emergencies. ${ }^{8}$ The present study also provided quantitative evidence supporting previous qualitative research. For example, Malagón-Rojas et a $2^{25}$ found that airport workers' perceived effectiveness of the COVID-19 prevention strategies (eg, disinfection of common areas and provision of hand sanitizers) facilitated a sense of security and bolstered workers' confidence in preventing the spread of infection at the airport. Another qualitative study found that individuals who were concerned about the impact of the pandemic on their health and income felt depressed and expressed negative attitudes towards the success of pandemic control. ${ }^{26}$ Consistent with these findings, our results have demonstrated that people's perceptions regarding the efficacy of prevention strategies may influence their attitudes towards the success of pandemic control through threat appraisals.

Considering the relatively small indirect effects in the current study, alternative mechanisms may be plausible. For example, research has demonstrated the essential role of self-efficacy in influencing individuals' attitudes towards the epidemic and the intentions for healthprotective behaviours, ${ }^{27} 28$ suggesting that self-efficacy may be an alternative mechanism underlying the association between perceived effectiveness of public health policies and attitudes towards pandemic control. Future research is needed to identify other potential mediators.

\section{Implications}

The study findings can guide the design and the implementation of health policies to facilitate people's confidence and psychological resilience during an infectious disease outbreak. The results demonstrated that individuals' threat appraisals may act as important pathways through which individuals' perceptions regarding public health measures may influence their attitudes towards the success of pandemic control. Thus, the health authorities should take measures to mitigate people's inappropriate threat appraisals and excessive health concerns when implementing prevention measures. Previous research has found that possessing accurate knowledge about an epidemic is associated with reduced health anxiety. ${ }^{3} 29$ Thus, combining the prevention strategies with health education programmes to improve basic knowledge of the disease may mitigate people's exaggerated severity estimates of the outbreak. ${ }^{30}$ In particular, establishing education programmes for the public to explain how the prevention strategies (eg, self-quarantine, hand hygiene and requirement for wearing face masks) can reduce the spread of the virus may increase people's perceived effectiveness of these strategies and improve compliance with the strategies, which may then facilitate their positive attitudes towards the battle against the pandemic. Moreover, offering emergency assistance to people with financial difficulties and providing remote medical consultation services to people with limited mobility may help alleviate the perceived threat of the pandemic, and build individuals' psychological resilience to get through the hard times. ${ }^{7}$ Besides, given that excessive health concerns sometimes deviate from rationality, ${ }^{31}$ and can lead to risk-taking behaviours and social disturbance during a public health crisis, ${ }^{32-34}$ mental health services (eg, cognitive-behavioural therapy) may be considered for people with excessive health concerns and illness severity overestimates. $^{35}$ 


\section{Limitations}

The study was subject to several limitations. First, the crosssectional design of our study impedes drawing conclusions of causal relationship or temporal precedence. In particular, our results suggested a good fit of the reverse mediation model, with individuals' positive attitudes mediating the relationship between perceived effectiveness of the public health measures and threat appraisals. However, Tate has pointed out that in mediation analysis, the conceptual time-ordering is the more fundamental criterion for selecting a mediator than the sequential time-ordering. ${ }^{36}$ That is, regardless of the temporal order of measurement, the predictor should occur before the mediator conceptually, and the mediator is supposed to precede the outcome variable conceptually. Based on the transactional model of stress and coping, ${ }^{56}$ individuals' beliefs that coping resources are available would lessen their perceived threat of the stressor, which then leads to perceived controllability of the stressful event. Thus, we believe the hypothesised model meets the conceptual time-ordering criterion. Future research with longitudinal or experimental design is needed to confirm our hypothesised mediation model. Second, measures of the variables were reliant on self-reports, which may be subject to response bias. For example, it is likely that some participants tended to select the highest response categories (acquiescent response style) or the middle response categories (midpoint response style) in the online survey. We cannot rule out the possibility that the observed outcome levels or associations among variables may be affected by these response biases. ${ }^{37}$ Third, females, the highly educated people and younger adults were overrepresented in the sample. In particular, the majority of our participants $(72.5 \%)$ were females, and $73.1 \%$ of the participants had a college-level education or above. Previous research has found that individuals' threat appraisals and psychological responses during public health events are associated with gender and educational level. ${ }^{27293839}$ Thus, whether our findings are generalisable to other populations warrants future research. Fourth, the sample was restricted to residents in Shanghai. The educational level and personal income level in Shanghai are relatively higher than many other cities in China. ${ }^{11}$ In addition, the severity of the COVID-19 outbreak and the implemented public health measures varied among different cities and countries. Thus, it calls for future studies to test the generalisability of our findings to other areas in China, and elsewhere. Finally, in order to reduce participant burden during the pandemic, we assessed the variables using self-developed questionnaires, and were not able to perform analyses on test-retest reliability and construct validity. These measures need to be further validated in future research.

\section{CONCLUSIONS}

The present study indicated that individual's perceptions of public health measures may influence their attitudes towards the success of pandemic control by influencing their threat appraisals, including concerns about contracting the virus, perceived impact of the pandemic on life and estimated duration of the pandemic. To facilitate people's positive attitudes during public health emergencies, the health authorities should take efforts to reduce people's inappropriate threat appraisals and excessive concerns when implementing prevention strategies.

\section{Author affiliations}

${ }^{1}$ School of Public Health, Shanghai Jiao Tong University School of Medicine, Shanghai, People's Republic of China

${ }^{2}$ Department of Biostatistics, University of Michigan, Ann Arbor, Michigan, USA ${ }^{3}$ The Center for Modern Chinese City Studies, East China Normal University, Shanghai, People's Republic of China

${ }^{4}$ School of Social Development, East China Normal University, Shanghai, People's Republic of China

${ }^{5}$ State Key Laboratory of Oncogenes and Related Genes, Center for Single-Cell Omics, School of Public Health, Shanghai Jiao Tong University School of Medicine, Shanghai, People's Republic of China

${ }^{6}$ Center for Health Technology Assessment, Shanghai Jiao Tong University China Hospital Development Institute, Shanghai Jiao Tong University, Shanghai, People's Republic of China

\section{Twitter Tian Gu @GuTian_TianGu}

Contributors YH (hypcyr@shsmu.edu.cn) and RW (rjwu@re.ecnu.edu.cn) contributed equally to the manuscript as corresponding authors and are the guarantors. QC, YH and RW conceived the study. QC and TG conducted analyses and led the writing. $\mathrm{AL}$ and $\mathrm{JC}$ managed the data acquisition and conducted literature search. HW and NL contributed to the analyses and results interpretation. All authors contributed to drafting and editing the manuscript. The corresponding author attests that all listed authors meet authorship criteria and that no others meeting the criteria have been omitted. All authors approved the final manuscript as submitted and agreed to be accountable for all aspects of the work.

Funding This work was supported by National Natural Science Foundation of China (grant numbers: 71874111, 81630086, 72004133), the National Key R\&D Program of China (grant number: 2018YFC2000700), Shanghai Pujiang Program (grant number: 2020PJC080) and Shanghai Jiao Tong University Scientific and Technological Innovation Funds (grant numbers: YG2020YQ01, YG2020YQ06).

\section{Competing interests None declared.}

Patient consent for publication Not required.

Ethics approval The study was conducted in accordance with the Declaration of Helsinki, and was approved by the ethics committee of Shanghai Jiao Tong University School of Medicine (protocol number: SJUPN-202002).

Provenance and peer review Not commissioned; externally peer reviewed.

Data availability statement Data are available upon reasonable request. All authors had full access to the data in the study. Sharing of the data of this project will be available on request.

Supplemental material This content has been supplied by the author(s). It has not been vetted by BMJ Publishing Group Limited (BMJ) and may not have been peer-reviewed. Any opinions or recommendations discussed are solely those of the author(s) and are not endorsed by BMJ. BMJ disclaims all liability and responsibility arising from any reliance placed on the content. Where the content includes any translated material, BMJ does not warrant the accuracy and reliability of the translations (including but not limited to local regulations, clinical guidelines, terminology, drug names and drug dosages), and is not responsible for any error and/or omissions arising from translation and adaptation or otherwise.

Open access This is an open access article distributed in accordance with the Creative Commons Attribution Non Commercial (CC BY-NC 4.0) license, which permits others to distribute, remix, adapt, build upon this work non-commercially, and license their derivative works on different terms, provided the original work is properly cited, appropriate credit is given, any changes made indicated, and the use is non-commercial. See: http://creativecommons.org/licenses/by-nc/4.0/. 


\section{ORCID iDs}

Qiao Chu http://orcid.org/0000-0003-3305-0875

Tian Gu http://orcid.org/0000-0002-0856-8843

\section{REFERENCES}

1 Taha S, Matheson K, Cronin T, et al. Intolerance of uncertainty, appraisals, coping, and anxiety: the case of the $2009 \mathrm{H} 1 \mathrm{~N} 1$ pandemic. Br J Health Psychol 2014;19:592-605.

2 Piltch-Loeb R, Merdjanoff AA, Bhanja A, et al. Support for vector control strategies in the United States during the Zika outbreak in 2016: the role of risk perception, knowledge, and confidence in government. Prev Med 2019;119:52-7.

3 Vielot NA, Stamm L, Herrington J, et al. United States travelers concern about Zika infection and willingness to receive a hypothetical Zika vaccine. Am J Trop Med Hyg 2018;98:1848-56.

4 Carbone EG, Echols ET. Effects of optimism on recovery and mental health after a tornado outbreak. Psychol Health 2017;32:530-48.

5 Lazarus RS, Folkman S. Stress, appraisal, and coping. New York: Springer, 1984

6 Folkman S, Lazarus RS, Dunkel-Schetter C, et al. Dynamics of a stressful encounter: cognitive appraisal, coping, and encounter outcomes. J Pers Soc Psychol 1986;50:992-1003.

7 Hanefeld J, Mayhew S, Legido-Quigley $\mathrm{H}$, et al. Towards an understanding of resilience: responding to health systems shocks. Health Policy Plan 2018;33:355-67.

8 Ma L, Christensen T, Trust G, et al. and Citizens' Risk Concerns: Evidence from Crisis Management in China. Public Performance \& Management Review 2019;42:383-404.

9 Prateepko T, Chongsuvivatwong V. Patterns of perception toward influenza pandemic among the front-line responsible health personnel in southern Thailand: a $Q$ methodology approach. BMC Public Health 2009;9:161.

10 Cheung C-K, Tse JW-L, C-k C. Institutional trust as a determinant of anxiety during the SARS crisis in Hong Kong. Soc Work Public Health 2008;23:41-54.

11 National Bureau of Statistics of China. Statistical Yearbook of China 2019, 2019.

12 Shanghai Municipal Statistics Bureau. Sample survey of $1 \%$ of population in Shanghai 2015. China Statistics Press, 2015.

13 Wu Z, McGoogan JM. Characteristics of and Important Lessons From the Coronavirus Disease 2019 (COVID-19) Outbreak in China: Summary of a Report of 72314 Cases From the Chinese Center for Disease Control and Prevention. JAMA 2020;323:1239-1242.

14 Siegrist M, Cvetkovich G. Perception of hazards: the role of social trust and knowledge. Risk Anal 2000;20:713-20.

15 Wang C, Pan R, Wan X, et al. Immediate psychological responses and associated factors during the initial stage of the 2019 coronavirus disease (COVID-19) epidemic among the general population in China. Int J Environ Res Public Health 2020;17. doi:10.3390/ijerph17051729. [Epub ahead of print: 06 Mar 2020].

16 Wang D, Hu B, Hu C, et al. Clinical characteristics of 138 hospitalized patients with 2019 novel coronavirus-infected pneumonia in Wuhan, China. JAMA 2020;323:1061.

17 Guan WJ, ZY N, Hu Y, et al. Clinical characteristics of coronavirus disease 2019 in China. N Engl J Med 2020.

18 Corp IBM. Ibm SPSS statistics for windows, version 24.0. Armonk NY: IBM Corp, 2016.

19 Muthén LK, Muthén BO. Mplus User's Guide. 7th edn. Los Angeles, CA: Muthén \& Muthén, 2016.
20 Fiedler K, Harris C, Schott M. Unwarranted inferences from statistical mediation tests - an analysis of articles published in 2015. J Exp Soc Psychol 2018;75:95-102.

21 Lt H, Bentler PM. Cutoff criteria for fit indexes in covariance structure analysis: conventional criteria versus new alternatives. Struct Equ Modeling 1999;6:1-55.

22 Mackinnon DP, Lockwood CM, Williams J. Confidence limits for the indirect effect: distribution of the product and resampling methods. Multivariate Behav Res 2004;39:99-128.

23 Chen N, Zhou M, Dong X, et al. Epidemiological and clinical characteristics of 99 cases of 2019 novel coronavirus pneumonia in Wuhan, China: a descriptive study. Lancet 2020;395:507-13.

24 Huang C, Wang Y, Li X, et al. Clinical features of patients infected with 2019 novel coronavirus in Wuhan, China. Lancet 2020;395:497-506

25 Malagón-Rojas J, Parra B EL, Mercado M. Infection and risk perception of SARS-CoV-2 among Airport workers: a mixed methods study. Int J Environ Res Public Health 2020;17. doi:10.3390/ ijerph17239002. [Epub ahead of print: 0312 2020].

26 Williams SN, Armitage CJ, Tampe T, et al. Public perceptions and experiences of social distancing and social isolation during the COVID-19 pandemic: a UK-based focus group study. BMJ Open 2020;10:e039334.

27 Scherr CL, Jensen JD, Christy K. Dispositional pandemic worry and the health belief model: promoting vaccination during pandemic events. J Public Health 2017;39:e242-50.

28 Corace KM, Srigley JA, Hargadon DP, et al. Using behavior change frameworks to improve healthcare worker influenza vaccination rates: a systematic review. Vaccine 2016;34:3235-42.

29 Zhong B-L, Luo W, Li H-M, et al. Knowledge, attitudes, and practices towards COVID-19 among Chinese residents during the rapid rise period of the COVID-19 outbreak: a quick online cross-sectional survey. Int J Biol Sci 2020;16:1745-52.

30 Legido-Quigley $\mathrm{H}$, Asgari N, Teo YY, et al. Are high-performing health systems resilient against the COVID-19 epidemic? Lancet 2020;395:848-50.

31 Taylor S, McKay D, Abramowitz JSJHoEBPiCP. Hypochondriasis and Health-Related anxiety 2012;2.

32 Ho CS, Chee CY, Ho RC. Mental health strategies to combat the psychological impact of COVID-19 beyond paranoia and panic. Ann Acad Med Singap 2020;49:1-3.

33 Jack A. Why the panic? South Korea's MERS response questioned. BMJ 2015;350:h3403.

34 Lima CKT, Carvalho PMdeM, Lima IdeAAS, et al. The emotiona impact of coronavirus 2019-nCoV (new coronavirus disease). Psychiatry Res 2020;287:112915.

35 Cooper K, Gregory JD, Walker I, et al. Cognitive behaviour therapy for health anxiety: a systematic review and meta-analysis. Behav Cogn Psychother 2017;45:110-23.

36 Tate CU. On the overuse and misuse of mediation analysis: it may be a matter of timing. Basic Appl Soc Psych 2015;37:235-46.

37 Stone AA, Schneider S, Junghaenel DU, et al. Response styles confound the age gradient of four health and well-being outcomes. Soc Sci Res 2019;78:215-25.

38 Mihashi M, Otsubo Y, Yinjuan X, et al. Predictive factors of psychological disorder development during recovery following SARS outbreak. Health Psychol 2009;28:91-100.

39 Bonanno GA, Ho SMY, Chan JCK, et al. Psychological resilience and dysfunction among hospitalized survivors of the SARS epidemic in Hong Kong: a latent class approach. Health Psychol 2008;27:659-67. 